\title{
The Critical Grid Size and Transmission Radius for Local-Minimum-Free Grid Routing in Wireless Ad Hoc and Sensor Networks
}

\author{
Chin-Wei YI ${ }^{1 *}$, Peng-Jun WAN $^{2}$, ChaO-Min Su${ }^{1}$ And Chen-Wei Huang ${ }^{1}$ \\ ${ }^{1}$ Department of Computer Science, National Chiao Tung University, Hsinchu City 30010, Taiwan \\ ${ }^{2}$ Department of Computer Science, Illinois Institute of Technology, Chicago, IL 60616, USA \\ ${ }^{*}$ Corresponding author: yi@cs.nctu.edu.tw
}

\begin{abstract}
In grid routing, the plane is tessellated into equal-sized square cells. Two cells are called neighbor cells if they share a common edge, and two nodes are called routing neighbors if they are in neighbor cells and within each other's transmission range. If communication parties are in the same cell, packets can be transmitted directly; otherwise, packets are forwarded to routing neighbors that are in cells closer to destination cells. As a greedy strategy, grid routing suffers the existence of local minima at which no neighbor nodes exist for relaying packets. To guarantee deliverability, in this paper, we investigate two vital parameters of grid routing, called the grid size and the transmission radius. Assume that nodes are represented by a Poisson point process with rate $n$ over a unit-area square, and let $l$ denote the grid size and $r$ the transmission radius. First, we show that if $l=\sqrt{\beta \ln n / n}$ for some constant $\beta$ and $r=\sqrt{5}$ l, then $\beta=1$ is the threshold for deliverability. In other words, there almost surely do not exist local minima if $\beta>1$ and there almost surely exist local minima if $\beta<1$. Next, for any given $\beta>1$, we give sufficient and necessary conditions to determine the critical transmission radius (CTR) for deliverability. Then, we show that as $\beta \cong 1.092$, the CTR $r \cong 2.09 \sqrt{\ln n / n}$ is the minimum over all $\beta>1$. Simulation results are given to validate this theoretical work.
\end{abstract}

Keywords: wireless ad hoc networks; wireless sensor networks; grid routing; local minima; geographic greedy routing; disk graphs; random deployment

Received 18 August 2009; revised 23 February 2010

Handing editor: Erol Gelenbe

\section{INTRODUCTION}

Wireless communication devices permeate our lives as technology advances and prices drop at the same time. People from both the industrial and research communities are highly motivated to explore the possible applications that utilize wireless connections over these autonomous devices to supplement or even eliminate the necessity of a pre-established network infrastructure. The devices form a self-organizing network and autonomously collaborate with each other to transport packets by serving as both end systems and routers at the same time. A communication session is established either through a single-hop radio transmission if the communication parties are close enough, or through relaying by intermediate devices otherwise. In some other applications, the ad hoc wireless devices may be dynamically switched to on or off or sometimes be in motion. For all these applications, it is natural to represent the ad hoc devices by a finite random point process over the deployment region. Consequently, the wireless ad hoc network is usually represented by a random graph.

The classic random graph model due to Erdôs and Rényi [1], in which each pair of vertices is joined by an edge independently and uniformly at some probability, is not suited to accurately represent networks of short-range radio nodes, due to the presence of local correlation among radio links. This motivated Gilbert [2] to propose an alternative random graph model for radio networks. Gilbert's model assumes that all devices, 
represented by an infinite random-point process over the entire plane, have the same maximum transmission radius $r$, and two devices are joined by an edge if and only if their distance is at most $r$. For the modeling of wireless ad hoc networks which consist of finite radio nodes in a bounded geographic region, a bounded (or finite) variant of the standard Gilbert model has been used by Gupta and Kumar [3] and others. In this variant, the random-point process representing the ad hoc devices is typically assumed to be a uniform $n$-point process over a disk or a square of unit area by proper scaling, and the wireless ad hoc network is exactly the $r$-disk graph, denoted by $G(n, r)$. To distinguish the random graph from the classic random graph due to Erdôs and Rényi, it is referred to as a random geometric graph.

The connectivity of a wireless ad hoc network is a fundamental requirement. For any constant $\xi$, Dette and Henze [4] showed that the graph $G\left(n, \sqrt{\frac{\ln n+\xi}{\pi n}}\right)$ has no isolated nodes with probability $\exp \left(-\mathrm{e}^{-\xi}\right)$ asymptotically. Penrose [5] established that if a random geometric graph has no isolated nodes, then it is almost surely connected. Gupta and Kumar [3] showed that the network $G\left(n, \sqrt{\frac{\ln n+c(n)}{\pi n}}\right)$ is asymptotically connected with probability one if and only if $c(n) \rightarrow \infty$ and disconnected with probability one if and only if $c(n) \rightarrow-\infty$. Goel et al. [6] showed that for random geometric graphs, all monotone graph properties, such as connectivity, have a sharp threshold and presented the upper bound on the threshold width. Instead of uniform deployment, Shakkottai et al. [7] considered an unreliable wireless sensor network with $n$ nodes, where the sensors are arranged in a grid over a square of unit area and found the necessary and sufficient conditions about the transmission radius of each sensor for coverage and connectivity.

Geographic-based routing algorithms for wireless ad hoc networks are proposed to lessen the maintenance burden of conventional topology-based routings by utilizing location information. The advancement of technologies in GPS and relative coordinate positioning systems using signal strength or topology information $[8,9]$ show the feasibility of applying geographic information in routing decisions. In this paper, we assume some location service system is available, for example, $[10,11]$. In [12], a survey on this topic is available. Also, Giordano and Stojmenovic [13] did a good survey on known routing methods and provided their taxonomy in terms of a number of characteristics.

On the basis of local knowledge of the network, greedy routing algorithms make decisions to obtain maximal benefit according to some greedy criteria. A local minimum occurs as no positive benefit can be obtained. Thus, packets cannot be delivered at local minima even if there exist paths between source and destination nodes. Most previous research works were with some remedial detour strategies when encountering local minima. An intuitive way is to select a node that leads to least retreat; however, this may introduce the looping packet problem which does not happen if only positive progress is allowed. Several face routing algorithms were proposed to handle the occurrence of local minima on an embedded planar graph [14-17]. However, as pointed out by Kim et al. [18], face routing algorithms may fail with realistic and non-idealized radios that do not comply with the unit-disk graph model. Leong et al. [19] proposed a geographical routing algorithm without planarization by constructing a spanning tree. Anyway, the simplest way is to discard the packet when no positive progress can be made [20]. This is applicable for real-time applications since late arrival of retransmission does not mean too much.

Since devices may join, leave the network or even move at will without prior notice, routing in such a dynamic environment has to be robust and scalable. Recent researches [11, 21] have shown that routing strategies, such as AODV [22], $D S D V$ [23] or DSR [24], without using geographical information in routing decisions, are not scalable. To further cope with the dynamic network topology, hierarchical structures were introduced to enhance scalability. Several two-level hybrid geographical routing strategies have been proposed. In the Terminode project [25], the proactive method is used for local traffic whereas greedy anchored geodesic forwarding is used for remote traffic. Some zone-based routing protocols were proposed separately by $\mathrm{Joa}-\mathrm{Ng}$ and $\mathrm{Lu}$ [26], Li et al. [27] and Liao et al. [28] by partitioning the network into fixedsize zones. For intra-zone traffic, traditional topology-based methods are used to enhance routing efficiency with affordable overhead. For inter-zone traffic, either link-state protocols or greedy geographic-based protocols can be used as demanded by different communication patterns. However, even if hierarchy is introduced into the greedy geographical routing algorithms, local minima still occur frequently.

As to the grid routing, Ye et al. [29] developed the two-tier data dissemination (TTDD) routing protocol, in which data is delivered by creating a virtual grid structure. Hornsberger and Shoja [30] proposed geographic grid routing based on TTDD to provide robust task dissemination and data collection. Wu et al. [31] described a new routing algorithm called the grid-based stable routing algorithm, in which extra stability information is contained in the routing.

In this work, we assume that wireless devices are represented by a Poisson point process with rate $n$ over a unit-area square region $\mathbb{D}$. Thus, a real-world deployment region in an application is properly scaled to a square of unit area. Each node has the same transmission radius $r_{n}$. In other words, we consider idealized radios. In grid routing, the plane is tessellated into equal-sized square cells; two cells are called neighbor cells if they share a common edge and two nodes are routing neighbors if they are in neighbor cells and within each other's transmission range. For a communication session, the cell that contains the source node is called the source cell and the cell that contains the destination node is called the destination cell. As one node sends or relays packets, if the destination node is in the same cell, packets are delivered directly to their destination; otherwise, packets are greedily forwarded to routing neighbors that are in 
cells closer to the destination cell measured by the Manhattan distance. Accordingly, we only consider the route horizontally or vertically. That only the last one hop may occur within the same cell can effectively reduce the time of relaying packets. Thus, a local minimum occurs as there is no routing neighbor for forwarding packets.

In such a scheme, there are two vital parameters to be decided, namely the grid size and the transmission radius. Instead of proposing a new remedial strategy, to guarantee the deliverability of packets, we seek to eliminate the existence of local minima via properly setting these two parameters. To put it another way, we eliminate the probability of existence of local minima by simply choosing a proper grid size and transmission radius. In particular, we will discuss three asymptotics from the probabilistic aspect, including the critical grid size that asymptotic almost surely (abbreviated to a.a.s.) guarantees that there does not exist an empty grid; the critical transmission radius $(C T R)$ that a.a.s. guarantees that there always exist relay nodes; and the smallest CTR over all grid size configurations. Our contributions are on finding the sufficient conditions for our goal and providing theoretical analysis and proof.

Gamal et al. [32] did great work in characterizing the delay and determining the throughput-delay trade-off in fixed and mobile ad hoc networks. They use cell area to parameterize the trade-off and figure out the optimal throughput-delay tradeoff. However, the focuses and approaches are different. Ours are for deliverability using scan statistics and theirs are for tradeoff using combinations of stochastic processes, queueing theory and so on

In what follows, the disk of radius $r$ centered at $x$ is denoted by $B(x, r)$. The unit-area square with its left lower corner at the origin is denoted by $\mathbb{D}$. An event is said to be a.a.s. if it occurs with a probability that converges to one as $n \rightarrow \infty$. The symbols $O, \Theta, \Omega, o$ and $\sim$ always refer to the limit $n \rightarrow \infty$. To avoid trivialities, we tacitly assume $n$ to be sufficiently large if necessary. In our simulations, $n=400$ is sufficient to obtain acceptable results. For simplicity of notation, the dependence of sets and random variables on $n$ will be frequently suppressed.

The rest of this paper is organized as follows. In Section 2, we present several useful geometric and probabilistic results. In Section 3, we derive the critical grid size and CTR for localminimum-free grid routing. In Section 4, simulation results are presented to verify our asymptotics. Finally, we summarize this paper in Section 5.

\section{PRELIMINARIES}

\subsection{Extremes of a collection of Poisson RVs}

First of all, we define a function $\phi(\mu)$ that will be frequently used in the subsequent discussion. Let $\phi(\mu)=1-\mu+$ $\mu \ln \mu$ for $\mu \in(0, \infty)$. A straightforward calculation yields $\phi^{\prime}(\mu)=\ln \mu$ and $\phi^{\prime \prime}(\mu)=1 / \mu$. Thus, $\phi$ is strictly convex and has the unique minimum zero at $\mu=1$ (see Fig. 1). Let

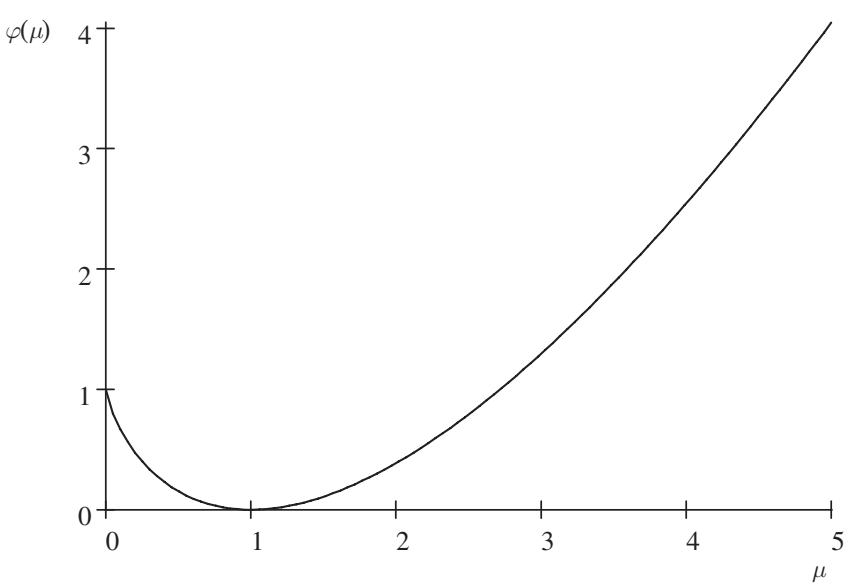

FIGURE 1. $\phi(\mu)=1+\mu \ln \mu-\mu$.

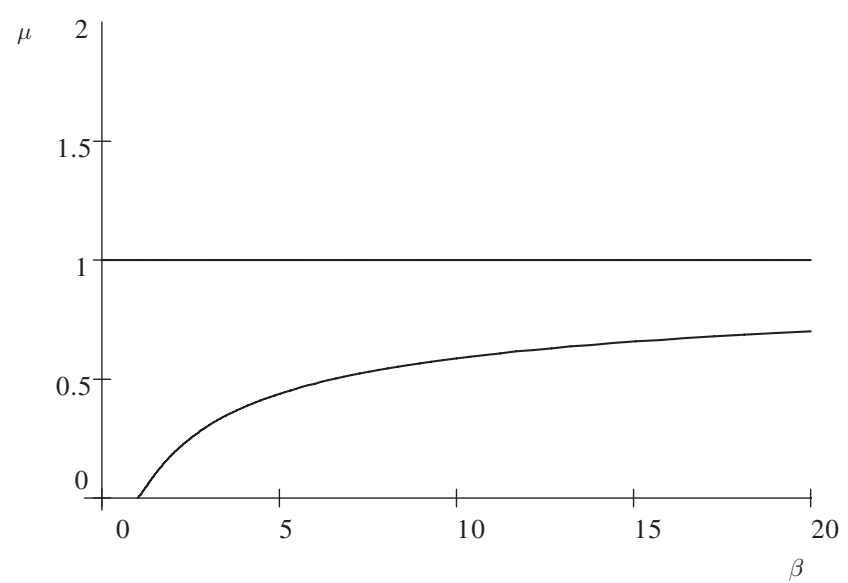

FIGURE 2. The curve is corresponding to $\mu=\phi_{-}^{-1}(1 / \beta)$. In addition, $\mu=1$ is the asymptotic line of $\mu=\phi_{-}^{-1}(1 / \beta)$.

$\phi_{-}^{-1}:[0,1) \rightarrow(0,1]$ be the inverse of $\phi$ with the restriction on $\phi$ 's domain to $(0,1]$, and we are interested in the function $\mu=\phi_{-}^{-1}(1 / \beta)$. Figure 2 is the curve of $\mu=\phi_{-}^{-1}(1 / \beta)$ in which the $x$-axis is of $\beta$ and the $y$-axis is of $\mu . \phi_{-}^{-1}(1 / \beta)=0$, when $0<\beta<1$. The next lemma gives an a.a.s. lower bound on the minimum of a collection of Poisson RVs [33].

Lemma 2.1. Assume that $\lim \lambda_{n} / \ln n=\beta$ for some $\beta>0$. Let $Y_{1}, Y_{2}, \ldots, Y_{I_{n}}$ be $I_{n}$ Poisson RVs with means $\lambda_{n}$.

(1) If $I_{n}=o(n \sqrt{\ln n})$, then for any $0<\mu<\phi_{-}^{-1}(1 / \beta)$, $\min _{i=1}^{I_{n}} Y_{i}>\mu \lambda_{n}$ a.a.s.

(2) If $Y_{1}, Y_{2}, \ldots, Y_{I_{n}}$ are independent and $I_{n}=\Omega(n / \ln n)$, then for any $\phi_{-}^{-1}(1 / \beta)<\mu<1, \min _{i=1}^{I_{n}} Y_{i} \leq \mu \lambda_{n}$ a.a.s.

According to Lemma $2.1, \beta \phi_{-}^{-1}(1 / \beta)$ could be an indicator of the threshold for $\min _{i=1}^{I_{n}} Y_{i}$, and actually, it is a guideline in the following discussion. For convenience, we define a function 


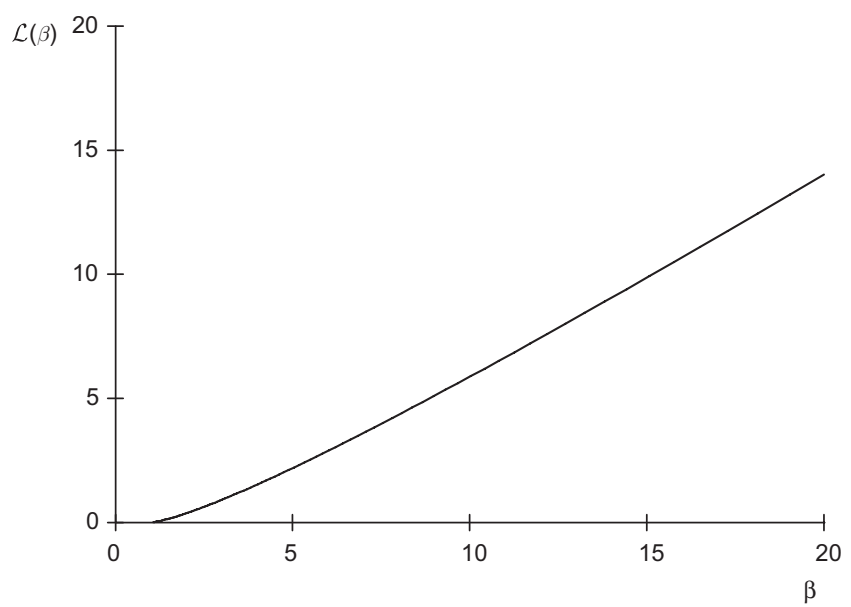

FIGURE 3. The curve $\mathcal{L}(\beta)$.

$\mathcal{L}$ over $(0, \infty)$ by

$$
\mathcal{L}(\beta)= \begin{cases}\beta \phi_{-}^{-1}(1 / \beta), & \text { if } \beta \geq 1, \\ 0, & \text { otherwise. }\end{cases}
$$

Here, $\mathcal{L}(\beta)$ is a monotonically the increasing function of $\beta$, and the curve of $\mathcal{L}$ is illustrated in Fig. 3.

\subsection{Scan statistics}

A useful theorem in scan statistics is going to be introduced to indicate the sufficient conditions about if there a.a.s. exist any nodes in a cell. Let $V \subset \mathbb{D}$ be a finite point set, and $C$ be a convex compact set. The minimum scan statistic for $V$ with scanning set $C$, denoted by $S_{m}(V, C)$, is defined as

$$
S_{m}(V, C)=\min _{C^{\prime} \cong C, C^{\prime} \subset \mathbb{D}}\left|V \cap C^{\prime}\right| .
$$

Here $C^{\prime} \cong C$ means that $C^{\prime}$ and $C$ are congruent. Let $\beta$ be a constant and $\mathbf{C}_{n}$ be a collection of convex compact sets such that, for any $C_{n} \in \mathbf{C}_{n},\left|C_{n}\right|=(\beta+o(1))(\ln n / n)$ and $\operatorname{diam}\left(C_{n}\right) \leq c_{0} \sqrt{\left|C_{n}\right|}$ for some constant $c_{0}$. Let $\mathcal{V}_{n}$ be a Poisson point process with rate $n$ over a unit-area square and $\mathcal{S}_{m}\left(\mathcal{V}_{n}, \mathbf{C}_{n}\right)=\min _{C_{n} \in \mathbf{C}_{n}} S_{m}\left(\mathcal{V}_{n}, C_{n}\right)$. The following theorem was given in [33].

THEOREM 2.1. For the asymptotics of $\mathcal{S}_{m}\left(\mathcal{V}_{n}, \mathbf{C}_{n}\right)$, it is almost sure that

$$
\operatorname{Pr}\left[\frac{\mathcal{S}_{m}\left(\mathcal{V}_{n}, \mathbf{C}_{n}\right)}{\ln n} \sim \mathcal{L}(\beta)\right] \rightarrow 1 .
$$

Furthermore, if $\beta<1$, we almost surely have

$$
\operatorname{Pr}\left[\mathcal{S}_{m}\left(\mathcal{V}_{n}, \mathbf{C}_{n}\right)=0\right] \rightarrow 1 .
$$

Thus, for any constant $\beta>1$, since $\mathcal{L}(\beta)>0$, Theorem 2.1 implies that any copy of $C_{n} \in \mathbf{C}_{n}$ contained in $\mathbb{D}$ a.a.s. covers at least one point of $\mathcal{V}_{n}$.

\section{CRITICAL GRID SIZE AND TRANSMISSION RADIUS}

In the grid routing, the plane is tessellated into equal-sized grids. Two cells are called neighbor cells if they share a common edge and two nodes are called routing neighbors if they are located in two neighbor cells and within each other's transmission range. In what follows, we assume nodes are represented by a Poisson point process with mean $n$ over the unit-area square region $\mathbb{D}$. Let $N=\lceil\sqrt{n / \beta \ln n}\rceil$ for some constant $\beta$ and $l=1 / N$. The deployment region $\mathbb{D}$ is divided into $N \times N$ equal-sized square cells with length $l$. Thus, the area of each cell is $l^{2}=(\beta+o(1))(\ln n / n)$, and the number of nodes in each cell is a Poisson RV with rate $n l^{2}=(\beta+o(1)) \ln n$. Each cell is given a grid coordinate $(i, j)$ where $1 \leq i, j \leq N$. We assume that each node knows the cell in which it is located by utilizing geographic information. The routing distance between two nodes is measured by the $L_{1}$ grid distance. In other words, if node $u$ is in the cell $\left(i_{u}, j_{u}\right)$ and node $v$ is in the cell $\left(i_{v}, j_{v}\right)$, the routing distances between them is given by $\left|i_{u}-i_{v}\right|+\left|j_{u}-j_{v}\right|$, the sum of vertical and horizontal distances between two cells measured by the number of cells. In each hop, except the last one to the destination node, packets are forwarded to routing neighbors with smaller routing distances to destination nodes, and thus, routing distances decrease by one. Local minima are encountered as none of the neighbors are with smaller routing distances to destination nodes.

In this section, we present the main results of this paper, namely the critical grid size and CTR. Note that the main idea is that we decide the critical grid size first, then achieve deliverability by tuning the transmission radius. For the critical grid size, if the constant $\beta$ is smaller than a critical value, we shall prove that there a.a.s. exist empty cells and some traffic need to cross these empty cells by grid routing, and thus, local minima cannot be avoided. On the other hand, if the constant $\beta$ is larger than a critical value, it is a.a.s. that every cell contains nodes. Hence, if we set the transmission radius not less than $\sqrt{5}$ times of the grid size, every node has routing neighbors in each of the four directions and, therefore, deliverability can be a.a.s. guaranteed. Furthermore, as the grid size is larger than the critical grid size, it is not necessary always to set the transmission radius as large as $\sqrt{5}$ times of the grid size. For a given tessellation, the CTR is the smallest transmission radius that guarantees that every node has at least one routing neighbor in each neighbor cell. We derive the CTR in the second subsection. In addition, for the sake of power saving, we also derive the minimum CTR over all grid sizes.

\subsection{The critical grid size}

In this subsection, we shall derive the critical grid size in terms of $\beta$. It is the most important parameter used in the tessellation.

THEOREM 3.1. If $\beta>1$ and $r=\sqrt{5}$ l, it is a.a.s. localminimum-free; if $\beta<1$, it is a.a.s. that there exist local minima. 
Proof. First, we consider the case in which $\beta>1$ and $r=\sqrt{5} l$. Let $Y_{1}, \ldots, Y_{N^{2}}$ denote the numbers of nodes in each cell. Since $N^{2}=\Theta(n / \ln n)$ and $Y_{1}, \ldots, Y_{N^{2}}$ are Poisson RVs with mean $(\beta+o(1)) \ln n$, according to Lemma 2.1(1), for $\phi_{-}^{-1}(1 / \beta)>0$,

$$
\lim _{n \rightarrow \infty} \operatorname{Pr}\left[\min _{1 \leq i \leq I_{n}} Y_{i}>0\right]=1 .
$$

Besides, recall that the area of each cell is $l^{2}=(\beta+$ $o(1))(\ln n / n)$. Thus, every cell a.a.s. contains at least one node by Theorem 2.1 . In addition, since $r=\sqrt{5} l$, every node has neighboring nodes in each of the four directions. Thus, the network is local-minimum-free.

Now, we consider the case in which $\beta<1$. For any constant $\beta<1$, we shall show that there a.a.s. exist some empty cells and some packets need to be delivered across these cells. See Fig. 4. For each horizontal and vertical strip of cells, we group the $2\lceil 1 / \beta\rceil$ cells at each end of the strip. This means that every single trip has two groups. There are $N$ horizontal strips and $N$ vertical strips. Thus, $I_{n}=4 N$. Let $Y_{1}, \ldots, Y_{I_{n}}$ denote the numbers of nodes in each group. Since $I_{n}=4 N=\Theta(\sqrt{n / \ln n})$ and $Y_{i}$ are Poisson RVs with rate $n\left(2\lceil 1 / \beta\rceil l^{2}\right)=(2+o(1)) \ln n$, according to Lemma 2.1(1), we have

$$
\lim _{n \rightarrow \infty} \operatorname{Pr}\left[\min _{1 \leq i \leq I_{n}} Y_{i}>0\right]=1 .
$$

In this case, $\lim \lambda_{n} / \ln n$ in Lemma 2.1 (1) becomes 2, and so $\mu \in\left(0, \phi_{-}^{-1}(1 / 2)\right)$.

By Theorem 2.1, each of $4 N$ groups a.a.s. contains at least one node. This implies that there may be traffic between two

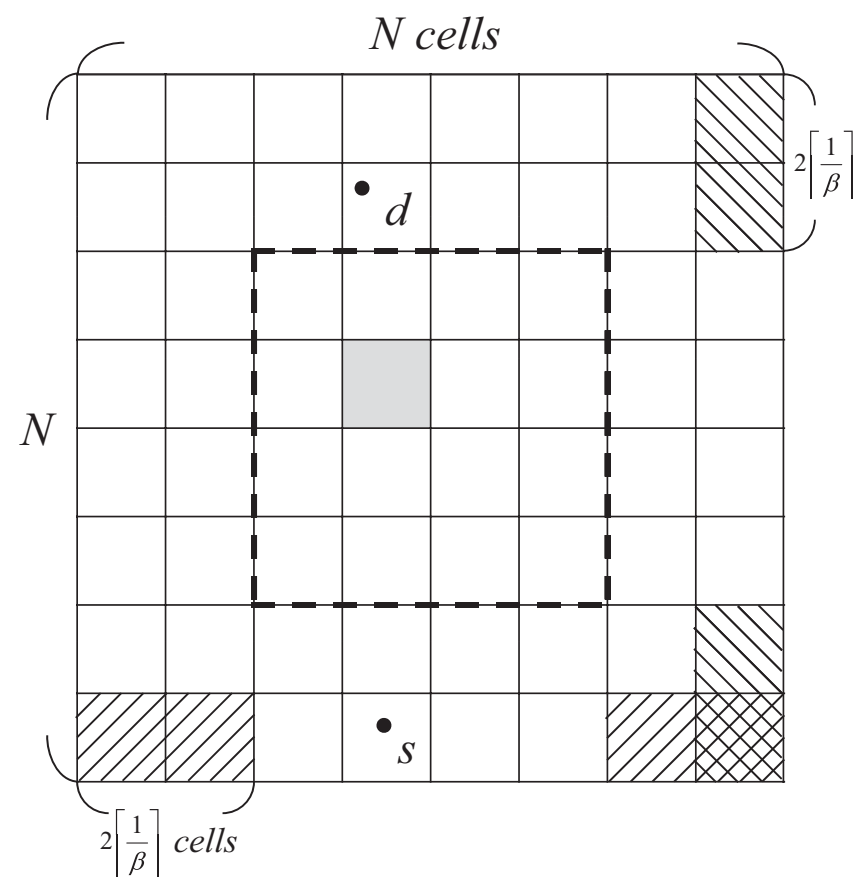

FIGURE 4. There are no nodes in the shaded cell that is in the routing path between nodes $s$ and $d$ in a unit-area deployment region. ends of each strip. Hence, if there are some empty cells in the middle, packets cannot be delivered across these empty cells. The number of remaining cells is $(N-4\lceil 1 / \beta\rceil)^{2}=\Theta(n / \ln n)$ and the number of nodes in each cell is an i.i.d. Poisson RV with rate $n l^{2}=(\beta+o(1)) \ln n$. In this case, $\lim \lambda_{n} / \ln n$ in Lemma 2.1(2) is $\beta$, and so $\mu \in(0,1)$. According to Lemma 2.1(2), by appropriately selecting $\mu$, we can conclude that there a.a.s. exist cells without nodes in them; that is,

$$
\lim _{n \rightarrow \infty} \operatorname{Pr}\left[\min _{1 \leq i \leq I_{n}} Y_{i}=0\right]=1
$$

Thus, the theorem is proved.

It is worth noting that the part of $\beta>1$ in Theorem 3.1, also included in [33], is a general result from minimum scan statistics. When $\beta<1$, it cannot be proved using minimum scan statistics.

\subsection{The CTR}

If every cell is not empty, in this subsection, we would like to know what is the smallest transmission radius that guarantees that every node has at least one routing neighbor in each neighbor cell. According to Theorem 3.1, in what follows, we only consider the tessellation with $\beta>1$. For a given $\beta>1$, we shall derive the CTR. Besides, note that packets are delivered between two neighbor cells in each hop, and thus the one-hop distance is at most $\sqrt{5} l$. Hence, it is not necessary to set the transmission radius larger than $\sqrt{5} l$, and we always implicitly assume that $r \leq \sqrt{5} l$.

For a given node, the covered neighboring region is the region of its neighbor cell covered by the transmission range. To avoid being a local minimum, there should exist nodes in each one of the four covered neighboring regions. Followed by Theorem 2.1, we know that if covered regions are not too slim, that is, not degenerated into shallow strips, the minimal number of nodes in the covered regions is only relevant to the area. As a node locates at a corner of a cell, the area in the opposite neighbor cell is minimal.

Assume that a node locates at a corner of a cell and let $A_{l}(r)$ be the region covered by the transmission range in a neighbor cell that does not share an edge with the corner. See Fig. 5. We should focus on conditions ensuring that $A_{l}(r)$ is non-empty which is sufficient to guarantee the non-emptiness of all the covered neighboring areas. Let $\left|A_{l}(r)\right|$ denote the area of the covered region and we have

$$
\left|A_{l}(r)\right|= \begin{cases}\frac{1}{2} r^{2} \arccos \frac{l}{r}-\frac{1}{2} l \sqrt{r^{2}-l^{2}}, & \text { if } l \leq r \leq \sqrt{2} l \\ \frac{1}{2} r^{2} \arcsin \frac{l}{r}+\frac{1}{2} l \sqrt{r^{2}-l^{2}}-l^{2}, & \text { if } \sqrt{2} l \leq r \leq 2 l \\ \frac{1}{2} r^{2}\left(\arcsin \frac{l}{r}-\arccos \frac{2 l}{r}\right)-l^{2} & \\ +\frac{1}{2} l\left(2 \sqrt{r^{2}-(2 l)^{2}}+\sqrt{r^{2}-l^{2}}\right), & \text { if } 2 l \leq r \leq \sqrt{5} l .\end{cases}
$$

The following theorem gives a threshold of the CTR. 


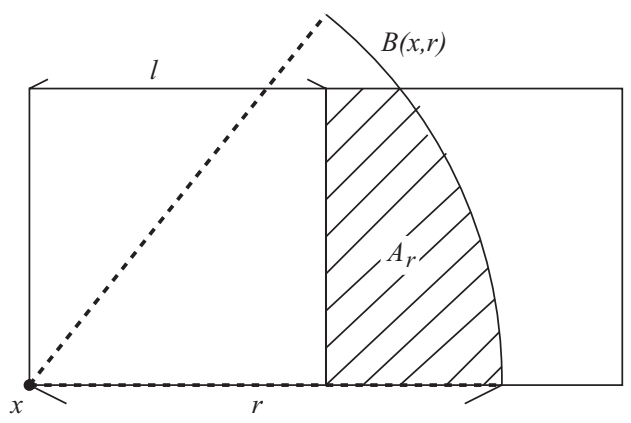

FIGURE 5. The lower bound of the covered neighboring area, $A_{r}$.

THEOREM 3.2. For any constant $\beta>1$, if the transmission radius $r$ is given by $\left|A_{l}(r)\right|=\alpha \ln n / n$ for some constant $\alpha$, then we have the following conditions.

(1) If $\alpha>1$, it is a.a.s. local-minimum-free.

(2) If $\alpha<1$, there a.a.s. exist local minima.

Proof. First, we prove that if $\alpha>1$, the network is a.a.s. localminimum-free. For a given grid size $l$ and transmission radius $r$, the area of the neighbor cell covered by the transmission range is minimum as the node locates at a corner opposite to the neighbor cell. The covered area in neighbor cells is at least $\alpha \ln n / n$. According to Theorem 2.1, for any constant $\alpha>1$, every node a.a.s. has neighboring nodes in each neighbor cell. Thus, it is a.a.s. that packets can be delivered in all directions, i.e. the network is a.a.s. local-minimum-free.

Now, we prove that if $\alpha<1$, there a.a.s. exist local minima. As $\beta>1$, according to Theorem 3.1, we know that there are some nodes in each cell a.a.s. Hence if we can show that there is any node without a neighbor in one of its neighbor cells, local minima will occur at this node when traffic is destinated to this particular neighbor cell. Choose $\varepsilon$, which is a constant for fixed $\alpha$, such that $\left|A_{l}(r+\sqrt{2} \varepsilon l)\right|<((1+\alpha) / 2)(\ln n / n)$. We consider the pairs of horizontal neighbor cells. Let $Y_{i}$ denote the joint event of the $i$-th pair that there are some nodes in the small square with width $\varepsilon l$ by the lower left corner of the left-hand-side cell, but there are no nodes in the covered area $A_{l}(r+\sqrt{2} \varepsilon l)$ of the right-hand-side cell. This event implies the occurrence of local minima at this pair of cells when there is traffic from nodes in the small square to nodes in the remaining portion of the right-hand-side cell. Let $A_{0}=(\varepsilon l)^{2}$ be the area of the small square. The probability of the event that some nodes exist in the small square is equal to

$$
1-\mathrm{e}^{-n A_{0}}=1-\mathrm{e}^{-n(\varepsilon l)^{2}} \rightarrow 1
$$

The probability of the event that there are no nodes in the covered area $A_{l}(r+\sqrt{2} \varepsilon l)$ of the right-hand-side cell is

$$
\mathrm{e}^{-n\left|A_{l}(r+\sqrt{2} \varepsilon l)\right|} \geq \mathrm{e}^{-n((1+\alpha) / 2)(\ln n / n)}=n^{-(1+\alpha) / 2} .
$$

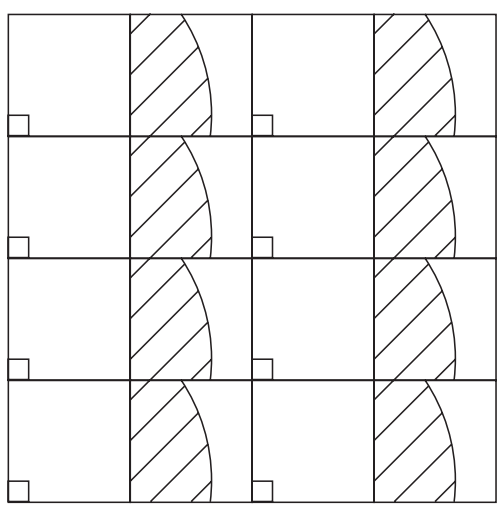

(b)

FIGURE 6. (a) $i$-th grid-pair with $X_{i}=1$, (b) all grid-pairs in $\mathbb{D}$.

Grouping two horizontal neighboring cells to a pair, we have $I_{n}=1 / 2 N^{2}=\Theta(n / \ln n)$ pairs. For $1 \leq i \leq I_{n}$, let $X_{i}$ be the indicator of the event that $Y_{i}$ occurs on the $i$-th pair of cells (see Fig. 6a), and $X=X_{1}+X_{2}+\ldots+X_{I_{n}}$ denote the total number of events that has occurred in the deployment region (see Fig. 6b). We prove that the event will occur by showing the probability of $X=0$ tends to 0 .

$$
\begin{aligned}
& \operatorname{Pr}(X=0)=\operatorname{Pr}\left(\sum_{i=1}^{I_{n}} X_{i}=0\right) \\
& =\prod_{i=1}^{I_{n}} \operatorname{Pr}\left(X_{i}=0\right)=\prod_{i=1}^{I_{n}}\left(1-\operatorname{Pr}\left(X_{i}=1\right)\right) \\
& \leq \prod_{i=1}^{I_{n}} \mathrm{e}^{-\operatorname{Pr}\left(X_{i}=1\right)}=\mathrm{e}^{-\sum_{i=1}^{I_{n}} \operatorname{Pr}\left(X_{i}=1\right)} .
\end{aligned}
$$

Here

$$
\begin{aligned}
& \sum_{i=0}^{I_{n}} \operatorname{Pr}\left(X_{i}=1\right) \geq I_{n}\left(1-\mathrm{e}^{-n A_{0}}\right) \mathrm{e}^{-\alpha \ln n} \\
& \quad=\Theta\left(\frac{n}{\ln n} e^{-\alpha \ln n}\right)=\Theta\left(\frac{n^{1-\alpha}}{\ln n}\right) \rightarrow \infty .
\end{aligned}
$$

Hence, $\operatorname{Pr}(X=0) \rightarrow 0$, and the event a.a.s. occurs. This implies that local minima a.a.s. exist.

\subsection{The minimal CTR}

In this subsection, our objective is to derive the minimal CTR over $\beta>1$, i.e. the smallest $r$ and its corresponding $\beta$ such that $\left|A_{l}(r)\right|=\ln n / n$. A simple corollary of Theorem 3.2 is that there a.a.s. exist local minima for $r<l$. Thus, $l<r<\sqrt{5} l$ is a reasonable constraint. 
In order to derive the minimal CTR, we first find a simple fact of the differential of $\left|A_{l}(r)\right|$. Let $\left|A_{l}(r)\right|=\ln n / n$, and consider $r$ to be a function of $l$. Applying implicit differentiation, we have

$$
\frac{\partial\left|A_{l}(r)\right|}{\partial r} \frac{\mathrm{d} r}{\mathrm{~d} l}+\frac{\partial\left|A_{l}(r)\right|}{\partial l}=0 .
$$

Since $\partial\left|A_{l}(r)\right| / \partial r$ is positive, we know that $\partial r / \partial l$ and $\partial\left|A_{l}(r)\right| / \partial l$ have opposite signs, and $\mathrm{d} r / \mathrm{d} l=0$ if and only if $\partial\left|A_{l}(r)\right| / \partial l=0$.

Next, we informally derive the partial deviation $\partial\left|A_{l}(r)\right| / \partial l$. Fix the transmission radius $r$ and enlarge the cell size by $\Delta l$; then $\left|A_{l}(r)\right|$ decreases $A_{\text {loss }}$ at the left but increases $A_{\text {gain }}$ at both the up and right side. The new covered area increases by $A_{\text {gain }}-A_{\text {loss. }}$. See Fig. 7. After some straightforward calculation, we obtain $\partial\left|A_{l}(r)\right| / \partial l$ as follows:

$$
\frac{\partial\left|A_{l}(r)\right|}{\partial l}= \begin{cases}-\sqrt{r^{2}-l^{2}}, & \text { if } l \leq r \leq \sqrt{2} l \\ \sqrt{r^{2}-l^{2}}-2 l, & \text { if } \sqrt{2} l \leq r \leq 2 l \\ \sqrt{r^{2}-l^{2}}-2 l & \\ +2 \sqrt{r^{2}-(2 l)^{2}}, & \text { if } 2 l \leq r \leq \sqrt{5} l\end{cases}
$$

If the area gained can compensate the area lost, that is, $A_{\text {gain }}>$ $A_{\text {loss }}$, then we can decrease the transmission radius $r$ and still have the new covered area at the critical size $\ln n / n$. After some calculation, we have

$$
\begin{aligned}
\frac{\partial\left|A_{l}(r)\right|}{\partial l}<0, \quad \text { as } r & \in\left[l, \frac{1}{3} \sqrt{3} \sqrt{\frac{65}{3}-\frac{8}{3} \sqrt{13} l}\right) \\
\frac{\partial\left|A_{l}(r)\right|}{\partial l}=0, \quad \text { as } r & =\frac{1}{3} \sqrt{3} \sqrt{\frac{65}{3}-\frac{8}{3} \sqrt{13}} l \text { and } \\
\frac{\partial\left|A_{l}(r)\right|}{\partial l}>0, & \text { as } r \in\left(\frac{1}{3} \sqrt{3} \sqrt{\frac{65}{3}-\frac{8}{3} \sqrt{13}} l, \sqrt{5} l\right] .
\end{aligned}
$$

Here $(1 / 3) \sqrt{3} \sqrt{(65 / 3)-(8 / 3) \sqrt{13}} \approx 2.004 \in[2, \sqrt{5}]$. Replacing $r$ by $2.004 l$ into the equation

$$
\begin{aligned}
\left|A_{l}(r)\right|= & \frac{1}{2} r^{2}\left(\arcsin \frac{l}{r}-\arccos \frac{2 l}{r}\right)-l^{2} \\
& +\frac{1}{2} l\left(2 \sqrt{r^{2}-(2 l)^{2}}+\sqrt{r^{2}-l^{2}}\right) \\
= & \frac{\ln n}{n},
\end{aligned}
$$

we obtain that the cell size corresponds to $\beta \cong 1.092$. Thus, the optimal configuration for the minimal CTR is

$$
\begin{gathered}
l \cong \sqrt{\frac{1.092 \ln n}{n}} \cong 1.045 \sqrt{\frac{\ln n}{n}}, \text { and } \\
r \cong 2.004 l \cong 2.094 \sqrt{\frac{\ln n}{n}} .
\end{gathered}
$$

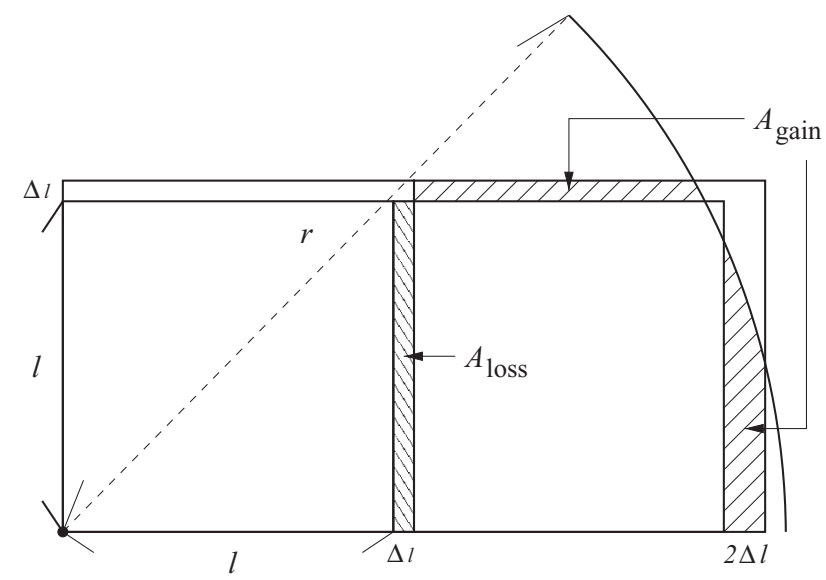

FIGURE 7. Covered neighboring area $A_{r}(r, l)$.

\section{SIMULATIONS}

To verify our asymptotic results, we executed extensive simulations on the critical grid size and CTR. In the simulation, 400, 800 and 1600 random points were generated with uniform distribution over a unit-area square, and 500 random point sets were generated for each size of sets. The deployment region is tessellated into $N \times N$ equal-sized square cells as before.

\subsection{The critical grid size}

First, we would like to verify the critical grid size. Intuitively, we may think if a tessellation is with no empty cell, then any tessellation with larger cell size is also with no empty cells; but this guess is not true. On the contrary, the critical grid size is not monotonic with respect to the partition number $N$. In other words, if a $N_{0} \times N_{0}$ tessellation is with no empty cell, it is not guaranteed that any $N \times N$ tessellation with $N<N_{0}$ is also with no empty cell. For example, in Fig. 8, for the same point set, there is no empty cell as $N=4$, but there exist some empty cells as $N=3$. For a given point set, we find out the largest empty square, which is an indication of the critical grid size, in $\mathbb{D}$ and let $l$ denote the length of the square. In Fig. 9, there are 100 random points and the small square is the corresponding largest empty square. Note that the critical cell size is within the range of $(1 / 2 l, l)$. The largest empty square problem can be solved by finding the largest empty circle in the Voronoi diagram constructed using the $L_{\infty}$ distance metric, and has a time complexity of $\Theta(n \log n)$ [34-36]. Hence, we will not delve into it further.

In Fig. 10, the vertical lines with labels T400, T800 and T1600 are our theoretical thresholds of critical cell sizes without empty cells with respect to $n=400, n=800$ and $n=1600$; and the curves from right to left are CDFs of the length of the largest empty square, $l$, with respect to $n=400, n=800$ and $n=1600$. According to the previous discussion, the critical 
(a)

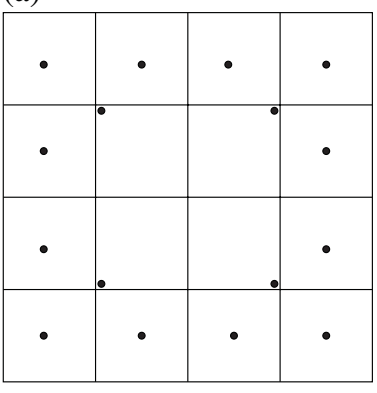

$N=4$ (b)

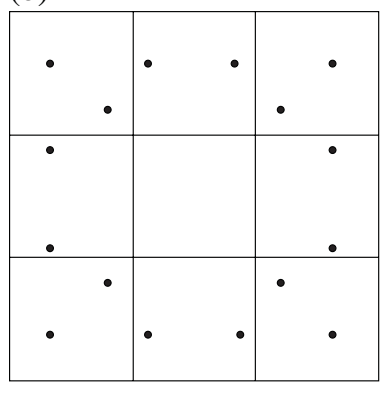

$N=3$

FIGURE 8. (a) As $N=4$, all cells are not empty; (b) As $N=3$, there exist empty cells.

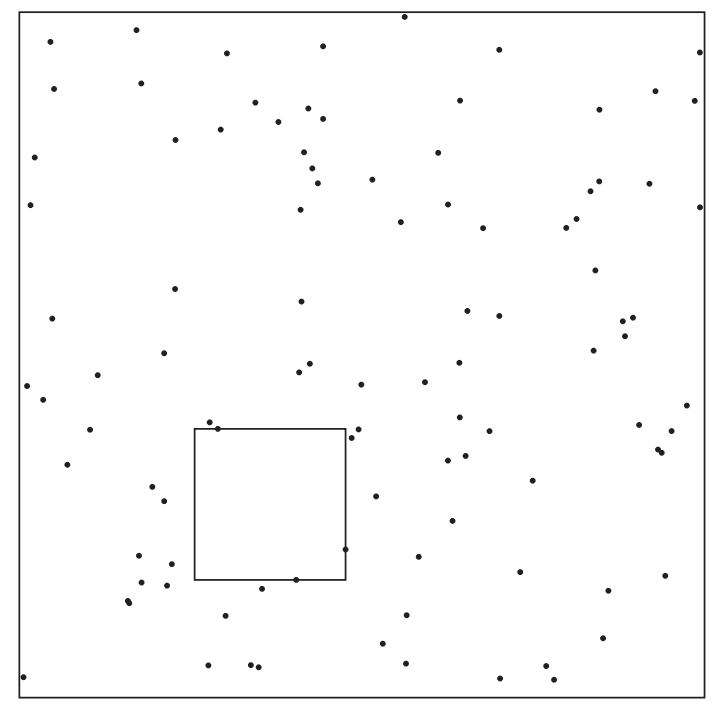

FIGURE 9. The largest empty square for $n=100$.

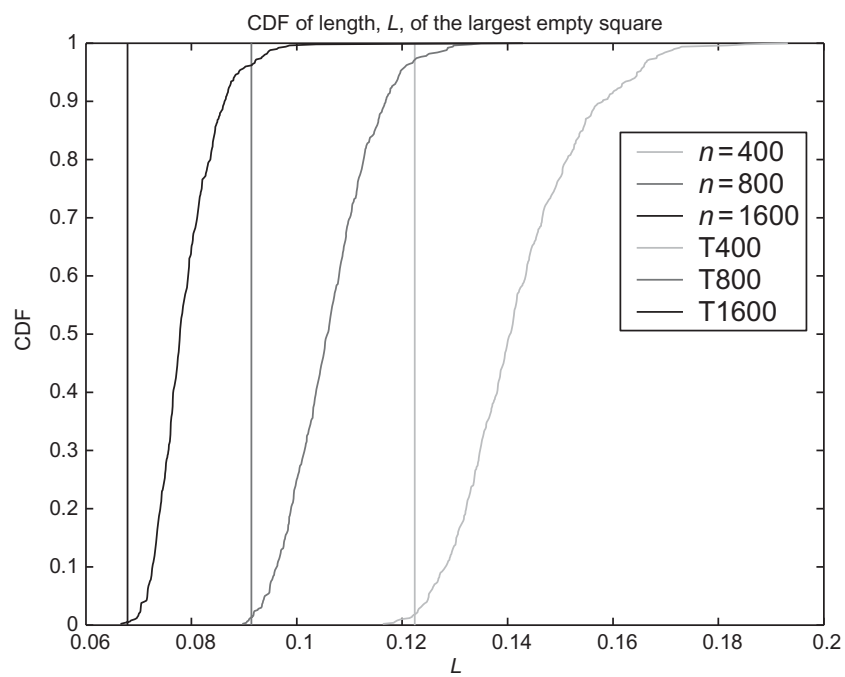

FIGURE 10. The CDF of the largest empty square length.

TABLE 1. Deviations between critical grid size and largest empty square.

\begin{tabular}{lll}
\hline Degree of deviation & $75 \%$ & $90 \%$ \\
\hline$n=400$ & $2.17 \%$ & $2.91 \%$ \\
$n=800$ & $2.19 \%$ & $2.78 \%$ \\
$n=1600$ & $2.07 \%$ & $2.71 \%$ \\
\hline
\end{tabular}

cell size is within the range $(1 / 2 l, l)$, and we can see that the theoretical value falls in this range in most cases.

Table 1 reveals the trend of convergence and we have listed the degree of deviation between the critical grid size and largest empty square length at CDF value of 75 and $90 \%$. We have observed that the error rate also decreases as the number of nodes increases since our derivation is based on asymptotic behavior, i.e. $n \rightarrow \infty$.

\subsection{The CTR}

Under the assumption that there is no empty cell in the deployment region $\mathbb{D}$, we are going to find out the minimum transmission radius that can guarantee the deliverability. As demanded by the grid routing, the transmission radius must be large enough to accomplish the following two conditions:

(i) Two nodes are directly reachable if they are located in the same cell.

(ii) Each node must be able to reach at least one neighbor among all of its neighboring cells.

For a given node $u$ in cell $(i, j)$, to avoid being a local minimum, it needs at least one routing neighbor in each of the four neighbor cells, and

$$
r(u)=\max \left\{\begin{array}{c}
\min _{v \in \operatorname{cell}(i, j+1)} d(u, v), \min _{v \in \operatorname{cell}(i, j-1)} d(u, v), \\
\min _{v \in \operatorname{cell}(i-1, j)} d(u, v), \min _{v \in \operatorname{cell}(i+1, j)} d(u, v)
\end{array}\right\}
$$

is the minimal transmission radius for $u$. Thus, the CTR is the maximum over the minimal transmission radius of each node in the deployment region, i.e. $\max _{u \in \mathbb{D}} r(u)$.

In Fig. 11, the $x$-axis, and $y$-axis, respectively, represent the grid size $L$ and transmission radius $r$. Since we consider the tessellation with $N=4,5, \ldots, 12$, the corresponding grid sizes are $L=\frac{1}{4}, \frac{1}{5}, \ldots, \frac{1}{12}$. The blue squares (respectively, green circles and red crosses) mark the critical transmission radii with $99 \%$ probability. The purple line is of $r=\sqrt{5} \mathrm{~L}$, which is an upper bound of the transmission radius guaranteeing the delivery between nodes in two neighbor cells. The simulation outcomes coincide with this. The blue line is of $r=\sqrt{2} L$, which is an upper bound of the transmission radius guaranteeing the delivery between nodes in the same cells. As the average number of nodes in a cell becomes large, the critical transmission radii may fall below this line. In such a case, the transmission radius for intra-cell transmission is larger than that for inter-cell 


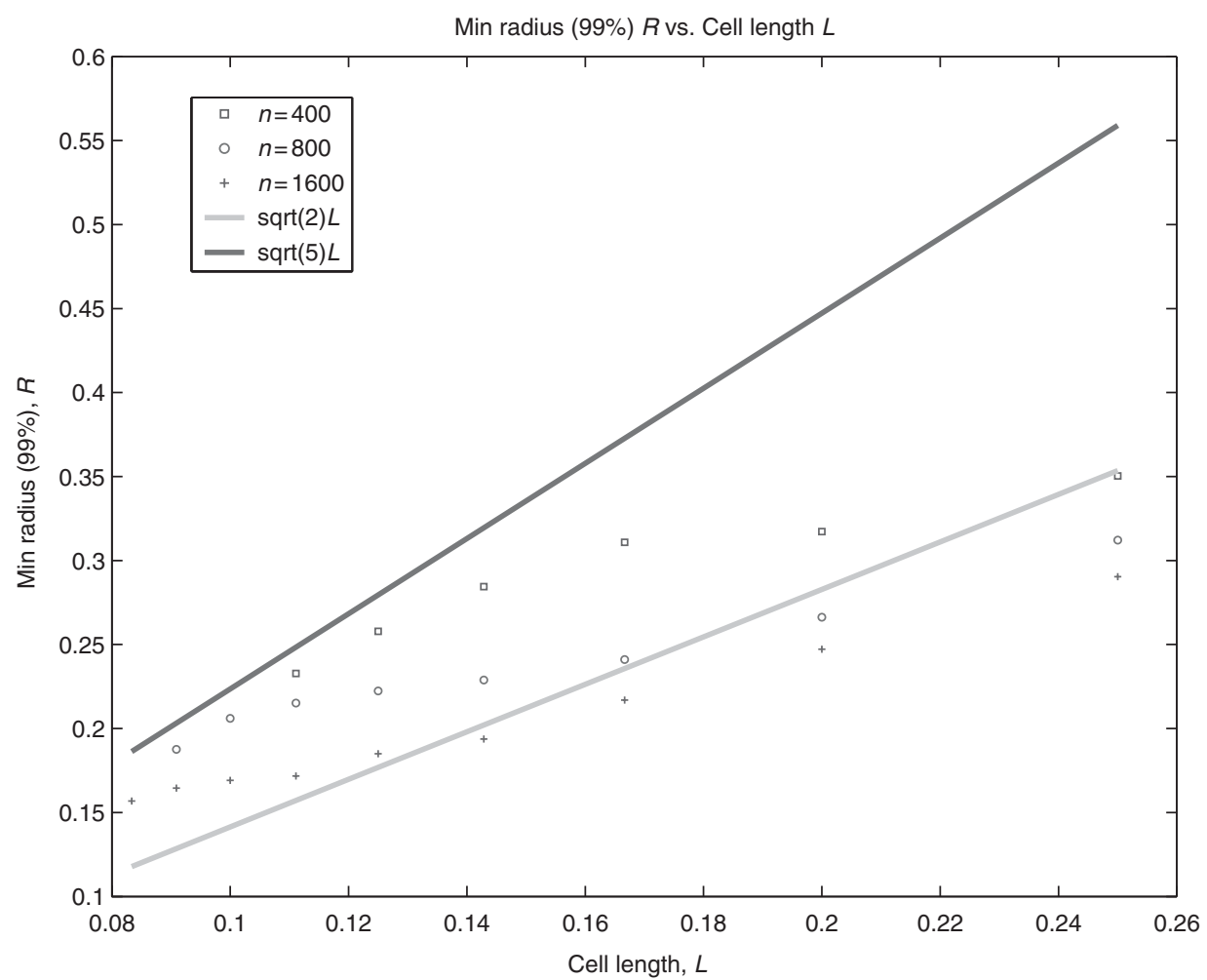

FIGURE 11. The minimum transmission radii for inter-cell delivery at $99 \%$.

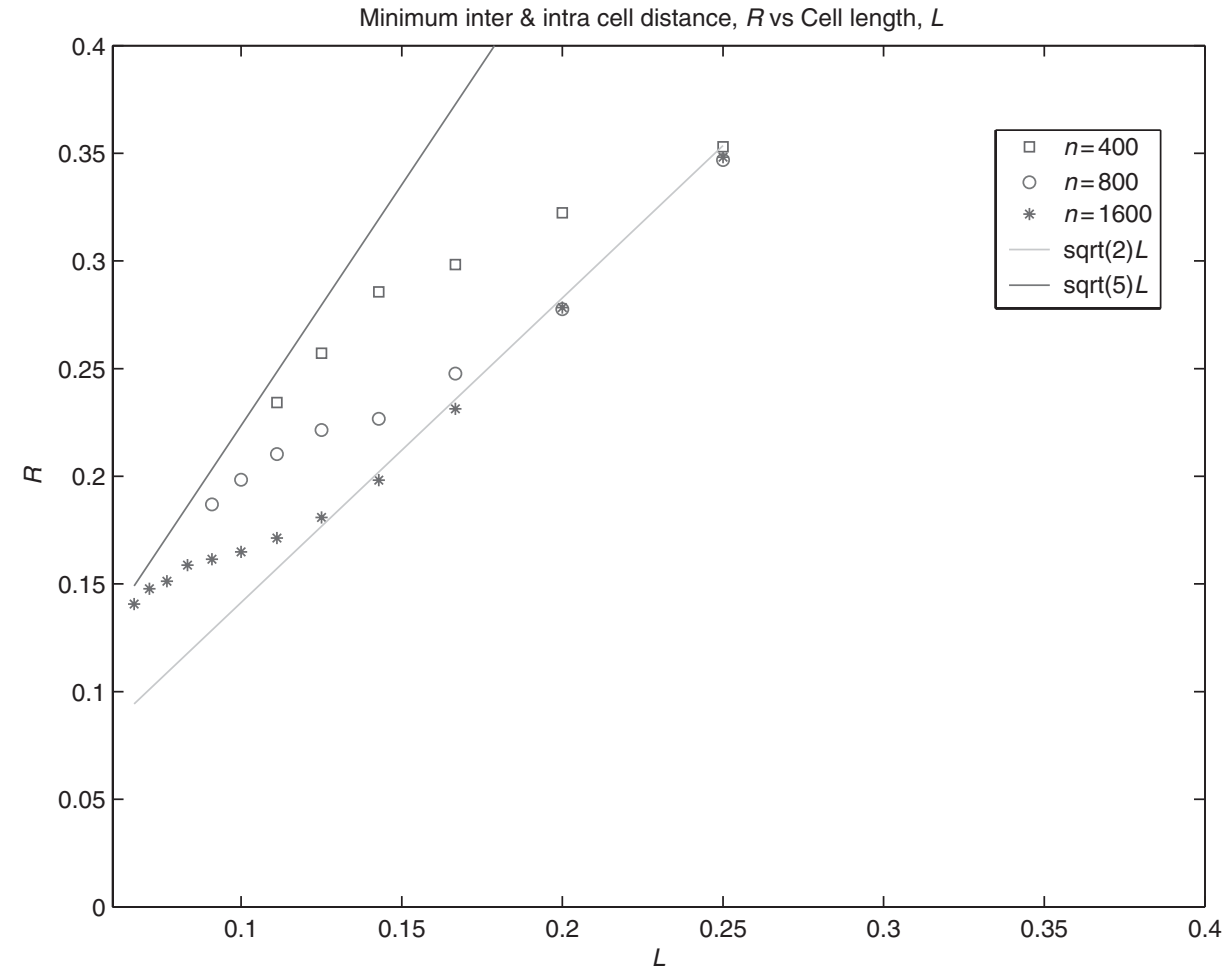

FIGURE 12. The minimum transmission radii for intra- and inter-cell delivery at $99 \%$ probability. 
transmission. Figure 12 is of the CTR after adding the intracell delivery as a consideration. Points below $r=\sqrt{2} L$ were uplifted. Again, this coincides with our analyzes.

\section{CONCLUSION}

Grid routing can be implemented in a localized, distributed and memoryless manner and is suitable to dynamic network environments. However, being a greedy algorithm, the grid routing suffers the local minima problem that hinders the greedy delivery of packets. In the literature, many remedial recovery strategies were proposed to handle the local minimum problem. In this paper, we eliminate the probability of existence of local minima by simply choosing a proper grid size and transmission radius. In addition to the critical grid size and CTR, we have also derived the minimal CTR guaranteeing packet delivery in favor of better power usage.

Among many mobility models in the literature, the random waypoint model is commonly used in ad hoc networks and is analyzed in depth by Bettstetter et al. [37] and Hyytiä et al. [38]. Future work will investigate the behaviors due to different mobility models. In this paper, we do not change the grid size dynamically. Changing the grid size dynamically may add some overhead to the network, but it can guarantee real-time deliverability. Therefore, it is interesting to find a better tradeoff between them.

\section{FUNDING}

This work of Dr. Yi described in this paper was partially supported by NSC under Grant No. NSC97-2221-E-009-052MY3 and NSC98-2218-E-009-023, by MoEA under Grant No. 98-EC-17-A-02-S2-0048, by ITRI under Grant No. 99-EC-17A-03-01-0809 and 99-EC-17-A-05-01-0626, and by the MoE ATU plan.

\section{REFERENCES}

[1] Erdős, P. and Rényi, A. (1960) On the evolution of random graphs. Publ. Math. Inst. Hung. Acad. Sci., 5, 17-61.

[2] Gilbert, E.N. (1961) Random plane networks. J. Soc. Ind. Appl. Math., 9, 533-543.

[3] Gupta, P. and Kumar, P.R. (1998) Critical Power for Asymptotic Connectivity in Wireless Networks. In McEneaney, W.M., Yin, G. and Zhang, Q. (eds.), Stochastic Analysis, Control, Optimization and Applications: A Volume in Honor of W. H. Fleming, March, pp. 547-566. Birkhauser.

[4] Dette, H. and Henze, N. (1989) The limit distribution of the largest nearest-neighbour link in the unit $d$-cube. J. Appl. Probab., 26, 67-80.

[5] Penrose, M.D. (1997) The longest edge of the random minimal spanning tree. Ann. Appl. Probab., 7, 340-361.

[6] Goel, A., Rai, S. and Krishnamachari, B. (2004) Sharp Thresholds for Monotone Properties in Random Geometric Graphs. Proc. 36th Annual ACM Symp. Theory of Computing (STOC '04), New York, NY, USA, June 13-15, pp. 580-586. ACM.

[7] Shakkottai, S., Srikant, R. and Shroff, N. (2003) Unreliable Sensor Grids: Coverage, Connectivity and Diameter. 22nd Annual Joint Conf. IEEE Computer and Communications Societies (INFOCOM '03), Vol. 2, pp. 1073-1083. San Francisco, CA, USA.

[8] Capkun, S., Hamdi, M. and Hubaux, J.-P. (2001) GPS-free Positioning in Mobile Ad-Hoc Networks. Proc. 34th Annual Hawaii Int. Conf. System Sciences (HICSS-34), January 3-6 9008. Maui, HI, USA.

[9] Rao, A., Ratnasamy, S., Papadimitriou, C., Shenker, S. and Stoica, I. (2003) Geographic Routing Without Location Information. Proc. 9th Annual Int. Conf. Mobile Computing and Networking (MobiCom '03), September 14-19, pp. 96-108. San Diego, CA, USA.

[10] Hubaux, J.-P., Boudec, J.-Y.L., Giordano, S. and Hamdi, M. (1999) The Terminode Project: Toward Mobile Ad-Hoc WANs. Proc. 6th IEEE Int. Workshop on Mobile Multimedia Communications (MOMUC'99), November 15-17, pp. 124-128. San Diego, CA, USA.

[11] Li, J., Jannotti, J., De Couto, D.S.J., Karger, D.R. and Morris, R. (2000) A Scalable Location Service for Geographic AdHoc Routing. Proc. 6th ACM Int. Conf. Mobile Computing and Networking (MobiCom '00), August, pp. 120-130. Boston, MA, USA.

[12] Hightower, J. and Borriella, G. (2001) Location systems for ubiquitous computing. IEEE Comput., 34, 57-66.

[13] Giordano, S. and Stojmenovic, I. (2002) Position-based Ad Hoc Routes in Ad Hoc Networks. In Ilyas, M. (ed.), The Handbook of Ad Hoc Wireless Networks, December 20, pp. 287-300. CRC Press, Inc., Boca Raton, FL, USA.

[14] Kranakis, E., Singh, H. and Urrutia, J. (1999) Compass Routing on Geometric Networks. Proc. 11th Canadian Conf. Computational Geometry, August, pp. 51-54. Vancouver, Canada.

[15] Karp, B. and Kung, H. (2000) GPSR: Greedy Perimeter Stateless Routing for wireless networks. Proc. 6th Annual Int. Conf. Mobile Computing and Networking (MobiCom '00), August 6-11, pp. 243-254. Boston, MA, USA.

[16] Bose, P., Morin, P., Stojmenović, I. and Urrutia, J. (2001) Routing with guaranteed delivery in ad hoc wireless networks. Wirel. Netw., 7, 609-616.

[17] Kuhn, F., Wattenhofer, R. and Zollinger, A. (2002) Asymptotically Optimal Geometric Mobile Ad-Hoc Routing. Proc. 6th Int. Workshop on Discrete Algorithms and Methods for Mobile Computing and Communications, September 28, pp. 24-33. ACM Press, Atlanta, GA, USA.

[18] Kim, Y.-J., Govindan, R., Karp, B. and Shenker, S. (2005) On the Pitfalls of Geographic Face Routing. Proc. 2005 Joint Workshop on Foundations of Mobile Computing (DIALM-POMC '05), September 2, pp. 34-43. ACM Press, Cologne, Germany.

[19] Leong, B., Liskov, B. and Morris, R. (2006) Geographic Routing Without Planarization. Proc. 3rd Conf. 3rd Symp. Networked Systems Design \& Implementation, May 8-10, pp. 339-352. San Jose, CA, USA.

[20] Wan, P.-J., Yi, C.-W., Yao, F.F. and Jia, X. (2006) Asymptotic Critical Transmission Radius for Greedy Forward Routing in 
Wireless Ad Hoc Networks. Proc. 7th ACM Int. Symp. Mobile Ad Hoc Networking and Computing (MobiHoc '06), New York, NY, USA, May 22-25, pp. 25-36. ACM.

[21] Jain, R., Puri, A. and Sengupta, R. (2001) Geographical routing using partial information for wireless ad hoc networks. IEEE Pers. Commun., 8, 48-57.

[22] Perkins, C., Belding-Royer, E. and Das, S. (2003). RFC 3561: Ad Hoc On-Demand Distance Vector (AODV) Routing, the Internet Engineering Task Force (IETF).

[23] Perkins, C. and Bhagwat, P. (1994) Highly Dynamic DestinationSequenced Distance-Vector routing (DSDV) for mobile computers. ACM Conf. Communications Architectures, Protocols and Applications (SIGCOMM '94), October, pp. 234-244. London, England, UK.

[24] Johnson, D., Hu, Y. and Maltz, D. (2007). RFC 4728: The Dynamic Source Routing Protocol (DSR) for Mobile Ad Hoc Networks for IPv4, the Internet Engineering Task Force (IETF).

[25] Blazevic, L., Buttyan, L., Capkun, S., Giordano, S., Hubaux, J.P. and Boudec, J.-Y.L. (2001) Self-organization in mobile ad-hoc networks: the approach of terminodes. IEEE Commun. Mag., 39, 166-174.

[26] Joa-Ng, M. and Lu, I.-T. (1999) A peer-to-peer zone-based twolevel link state routing for mobile ad hoc networks. IEEE J. Sel. Areas Commun., 17, 1415-1425.

[27] Li, Q., Aslam, J.A. and Rus, D. (2001) Online Power-Aware Routing in Wireless Ad-Hoc Networks. Proc. 7th Annual Int. Conf. Mobile Computing and Networking (MobiCom '01), July, pp. 97-107. Rome, Italy.

[28] Liao, W.-H., Sheu, J.-P. and Tseng, Y.-C. (2001) GRID: A fully location-aware routing protocol for mobile ad hoc networks. Telecommun. Syst., 18, 37-60.

[29] Ye, F., Luo, H., Cheng, J., Lu, S. and Zhang, L. (2002) A Two-Tier Data Dissemination Model for Large-Scale Wireless Sensor
Networks. Proc. 8th Annual Int. Conf. Mobile Computing and Networking (MobiCom '02), New York, NY, USA, pp. 148-159. ACM.

[30] Hornsberger, J. and Shoja, G.C. (2005) Geographic Grid Routing for Wireless Sensor Networks. Proc. IEEE Int. Conf. Networking, Sensing and Control (ICNSC '05), March 19-22, pp. 484-489. Tucson, AZ, USA.

[31] Wu, Z., Song, H., Jiang, S. and Xu, X. (2007) A Grid-based Stable Routing Algorithm in Mobile Ad Hoc Networks. Proc. 2007 First Asia Int. Conf. Modelling and Simulation (AMS '07), May 27-30, pp. 181-186. Phuket, Tailand.

[32] Gamal, A.E., Mammen, J., Prabhakar, B. and Shah, D. (2004) Throughput-Delay Trade-Off in Wireless Networks. 23rd Annual Joint Conf. IEEE Computer and Communications Societies (INFOCOM '04), March 7-11, pp. 475-486. IEEE, Hong-Kong, China.

[33] Yi, C.-W. (2009) A unified analytic framework based on minimum scan statistics for wireless ad hoc and sensor networks. IEEE Trans. Parallel Distrib. Syst. (TPDS), 20, 1233-1245.

[34] Hwang, F.K. (1979) An $O(n \log n)$ algorithm for rectilinear minimal spanning trees. J. Assoc. Comput. Mach., 26, $177-182$.

[35] Lee, D. and Wong, C. (1980) Voronoi diagrams in $L_{1}\left(L_{\infty}\right)$ metrics with 2-dimensional storage applications. SIAM J. Comput., 9, 200-211.

[36] Naamad, A., Lee, D. and Hsu, W. (1984) On the maximum empty rectangle problem. Discrete Appl. Math., 8, 267-277.

[37] Bettstetter, C., Resta, G. and Santi, P. (2003) The node distribution of the random waypoint mobility model for wireless ad hoc networks. IEEE Trans. Mob. Comput., 2, 257-269.

[38] Hyytiä, E., Lassila, P. and Virtamo, J. (2006) Spatial node distribution of the random waypoint mobility model with applications. IEEE Trans. Mob. Comput., 5, 680-694. 